\title{
The Relationship Between Perceived Barriers for Physical Activity and Depression in Low Active Community-Dwelling Women
}

Caroline Figueroa ( $\nabla$ c.a.figueroa@berkeley.edu )

University of California, Berkeley

\section{Adrian Aguilera}

University of California, Berkeley

Thomas Hoffmann

University of California, San Francisco

Yoshimi Fukuoka

University of California, San Francisco

\section{Research Article}

Keywords: Physical Fitness, Depression, Women's Health, Preventive Medicine, Exercise

Posted Date: January 12th, 2021

DOI: https://doi.org/10.21203/rs.3.rs-135211/v1

License: (a) (1) This work is licensed under a Creative Commons Attribution 4.0 International License.

Read Full License 


\section{Abstract}

Background: Women are less physically active, report greater perceived barriers for exercise and show higher levels of depression. This contributes to high global disability. The relationship between perceived barriers for physical activity and depressive symptoms in women remains largely unexplored. The aims of this cross-sectional analysis were to examine the association between physical activity barriers and depressive symptoms, and identify types of barriers in low active community dwelling women.

Methods: 318 physically inactive women aged 25-65 years completed the Barriers to Being Active Quiz (BBAQ) developed by the Centers for Disease Control and Prevention, and the Center for Epidemiological Studies Depression Scale (CES-D) at the baseline visit of the mobile phone based physical activity education (mPED) trial. The BBAQ consists of 6 subscales (lack of time, social influence, lack of energy, lack of willpower, fear of injury, lack of skill, and lack of resources). We used multivariate regression analyses, correcting for sociodemographics.

Results: Higher physical activity barriers were associated with greater depression scores (linear effect, estimate $=0.75,95 \% \mathrm{Cl}: 0.39-1.12, \mathrm{p}<0.001)$. This effect appeared to taper off for the higher barrier scores (quadratic effect, estimate: $-0.02,95 \% \mathrm{Cl}:-0.03--0.01, p=0.002$ ). Exploratory analyses indicated that score differences were most driven by the social influence $(p=0.027)$ and lack of energy subscales $(p=0.017)$.

Conclusions: Higher depression scores were associated with higher physical activity barriers. Social influence and lack of energy were particularly important barriers. Thus, addressing these barriers may improve the efficacy of physical activity interventions in women with higher depressive symptoms. However, a randomized controlled trial is warranted.

Trial Registration ClinicalTrials.gov\#: NCT01280812 registered January 21, 2011

\section{Introduction}

Despite the physical and mental health benefits of regular physical activity, women in every age group are less likely to meet the recommended levels of physical activity than men(1) (2) (3). According to both self-report and accelerometry measures, moderate and vigorous physical activity (MVPA) levels were higher in men than women(1). This may be in part because women face greater barriers to barriers to exercise than men(4) (5). Common self-reported barriers in women include lack of time and motivation(3), the failure to see themselves as athletes(4), and health problems(6) (7).

Women also have a risk up to two-fold compared to men of developing depression(8). Evidence from Mendelian randomization studies suggests that physical inactivity and depression have a bidirectional relationship(9). Those diagnosed with depression are less likely to maintain an exercise program(10). In turn, increasing physical activity can reduce depressive symptoms(11), and prevent depressive episodes(12). Therefore, focusing on promoting physical activity in women can substantially benefit both 
women's mental (e.g. reduce depressive symptoms) and physical health (e.g. reduce the risk of diabetes, cardiovascular disease and cancer)(13).

In depression, a scoping review found that emotional constraints, which included "low mood", "lack of energy" and "being too tired" were among the most important barriers for physical activity(14). However, less is known about the relationship between physical activity barriers and depression in physically inactive women. This information would be highly beneficial, as barriers to physical activity may moderate the effect of physical activity interventions(15). Physical activity interventions have been designed both specifically for women(16) and for depression(17). Though these interventions have shown medium to high effect sizes (standardized mean difference of 0.28 after 6 months(18)), they may suffer from reduced effectiveness if they don't tailor specifically to individual barriers and mental health symptoms.

It's imperative to understand if women with higher depressive symptoms report higher barriers, and which unique barriers are of particular importance. This will help us to better tailor physical activity interventions to this important subgroup.

This paper examines the relationship between self-report physical activity barriers and depressive symptoms in community dwelling physically inactive women who enrolled in a mobile physical activity intervention, the mobile phone based physical activity education (mPED).

\section{The aims of this study were to:}

1) Examine if depressive symptoms predict the total barriers to physical activity score, correcting for demographics, clinical variables and social support.

2) Assess if depressive symptoms predict the barrier subscale scores correcting for demographics, clinical variables and social support in an exploratory manner.

\section{Methods}

\section{Study design and sample}

In this cross-sectional analysis of 318 women in the MPED trial, we analyzed the sociodemographic, clinical, and self-reported questionnaire data collected the screening/baseline study visit. Detailed descriptions of the study design and outcomes have been previously published(19)'(20)'(21) $(20,22,23)$. In short, eligibility criteria were female sex, age from 25 to 65 years, body mass index (BMl; calculated as weight in kilograms divided by height in meters squared) of 18.5 to 43.0, physically inactive at work and/or during leisure time based on the Stanford Brief Activity Survey, intent to be physically active, access to a home telephone or mobile phone, ability to speak and read English, no medical conditions or physical problems that required special attention in an exercise program, no current participation in other lifestyle modification programs, and no mild cognitive impairment as determined by the Mini-Cog test. 
During the screening/baseline visit, sociodemographics, medical and lifestyle history, the Center for Epidemiological Studies Depression Scale (CES-D)(24), body mass index (BMI) and the Barriers to Being Physically Active Quiz (BBAQ) were assessed by a trained research staff. All methods were conducted in accordance with the Declaration of Helsinki.

\section{Measures}

\section{Depressive symptoms}

The Center for Epidemiological Studies Depression Scale (CES-D)(24) was used to assess self-reported depressive symptoms. The CES-D is a valid and reliable instrument that is widely used to assess depressive symptoms in a research context. The CESD ranges from (0-60), with a cutoff score of 16 indicating risk for clinical depression.

Physical activity barriers scale: The Barriers to Being Physically Active Quiz developed by the Centers for Disease Control and Prevention (CDC)(25) is a 21-item measure assessing the following barriers to physical activity: 1) lack of time, 2) social influence, 3) lack of energy, 4) lack of willpower, 5) fear of injury, 6) lack of skill, and 7) lack of resources (e.g., recreational facilities, exercise equipment). Each domain contains 3 items, with a total score range of 0 to 63 . Respondents rate the degree of activity interference on a 4-point scale, ranging from 0 = "very unlikely" to 3 = "very likely."

\section{Other measures}

A research staff asked the participants to fill out the sociodemographic and medical questionnaires immediately after obtaining the written consent form. The emotional support question, "How many people can you count on to provide you with emotional support?" was developed by the research team. To calculate body mass index (BMI), weight was measured with a Tanita WB-110 digital electronic scale, and height was measured at baseline with a standard stadiometer.

\section{Statistical analysis}

We assessed the relationship between depressive symptoms and the total Barriers to Being Physically Active Quiz scale score correcting for demographics, clinical variables and social support. We additionally conducted a posthoc analysis to examine the relationship between depression and the BBAQ subscales.

We used descriptive statistics to summarize sample characteristics and linear and logistic regression to examine the association between the total barriers and subscale scores with depression scores. For subscale scores with a non-normal distribution we computed a binary variable based on the median scores. We included self-reported age, BMI, employment (paid work, yes or no), whether participants have children at home (yes/no), whether they have driven a car in the past week (yes/no) and marital status as covariates in the model, based on previous evidence(19),(20)'(21) (26). We examined non-linear effects of depression by including the quadratic effect of depression in the models and used Likelihood Ratio (LR) tests to assess the need for including these non-linear effects. We removed influential observations using 
Cook's d (see results; we also conduct a sensitivity test when the influential observations are not removed) and checked further model assumptions by visual inspections of residual plots. Tables are presented in the supplementary analysis. Analyses were carried out in R studio V. 1.1.423.

\section{Results}

Baseline characteristics of the 318 participants and the mean or median scores of the total barriers and the subscales are shown in Table 1. For the overall sample, based on the mean and median scores, the greatest barriers were lack of time, lack of energy and lack of willpower. Fear of injury was the least common reported barrier. In the supplementary material (Supplementary Table 1), we also show differences in the total scale and subscales between groups with high depression (CES-D $\geq 16$ ) for illustrative purposes (in analyses we used the continuous scores). 
Table 1

Baseline sample characteristics ( $N=318)$

Mean ( \pm SD), median ( IQR; not normally distributed variables) or $\mathrm{n}(\%)$

\section{Age}

Median

$54.0(50 / 60 / 60)$

Ethnicity

Native Hawaiian/Pacific Islander $1(0.3 \%)$

Black/African-American $26(8.2 \%)$

Hispanic/Latino $20(6.3 \%)$

Asian $\quad 65(20.4 \%)$

White (non-Hispanic) 179 (56.3\%)

More than 1 race $27(8.5 \%)$

\section{Education}

Completed High School \& some $\quad 77$ (24.2\%)

college

Completed College

$128(40.3 \%)$

Completed Graduate School

$113(35.5 \%)$

Annual household income

Under $\$ 40,000$

$62(19.5 \%)$

$\$ 40,001$ to 75,000

$77(24.2 \%)$

Over $\$ 75,000$

$153(48.1 \%)$

Don't know or Declined to state

$26(8.2 \%)$

Marital status

Never married

$99(31.1 \%)$

Currently married/cohabitating

$158(49.7 \%)$

Divorced/widowed

$61(19.2 \%)$

Employment

No, Full or Part time Job No Shift 160 (50.3\%) Work

$\mathrm{BBAQ}=$ the Barriers to Being Active Quiz. 
Mean ( \pm SD), median ( IQR; not normally distributed variables) or $\mathrm{n}(\%)$

Yes Full or Part time Job with Shift $71(22.3 \%)$

Work

No paid employment

$87(27.4 \%)$

Antidepressant

No

$246(77.4 \%)$

Yes

$72(22.6 \%)$

Emotional support

Support from $\geq 3$ people

$257(80.8 \%)$

Body mass index $\left(\mathrm{kg} / \mathrm{m}^{2}\right)$

Mean (SD)

$29.6(6.13)$

Self-reported high blood pressure (\%)

No

$232(73.0 \%)$

Yes

$80(25.2 \%)$

Don't know

$6(1.9 \%)$

Self-reported high cholesterol (\%)

No

$175(55.0 \%)$

Yes

97 (30.5\%)

Don't know

$46(14.5 \%)$

Barriers scale and subscales

BBAQ Sum score

$25(17 / 25 / 31)$

Lack of time

$4.23(2.68)$

Social influence

$3.53(2.10)$

Lack of energy

$4.18(2.64)$

Willpower

$7.00(6 / 7 / 8)$

Fear of injury

$0.00(0 / 0 / 2)$

Lack of skills

$1.00(0 / 1 / 3)$

Lack of resources

$2.00(0 / 2 / 4)$

$\mathrm{BBAQ}=$ the Barriers to Being Active Quiz. 


\section{The Barriers to Being Physically Active Quiz Total Score}

For the total Barriers to Being Physically Active Quiz scale score, a model that included a quadratic term was a better fit than a model without this term (of note a cubic term was not significant, $p=0.09$ ). We removed 4 influential observations (Cook's $d$ ). The total barriers scale showed a positive significant relationship with depression (linear effect of depression, estimate $=0.75,95 \% \mathrm{Cl}: 0.39-1.12, \mathrm{p}<0.001$ ), which tapered off for the higher barrier scores (quadratic effect of depression, estimate: $-0.02,95 \% \mathrm{Cl}$ : $-0.03--0.01, p=0.002$ ). In all models we corrected for self-reported age, BMI, employment (paid work, yes or no), whether participants have children at home (yes/no), whether they have driven a car in the past week (yes/no) and marital status. When influential observations were retained in the model in a sensitivity analysis, results were similar for the linear effect (linear effect of depression, estimate $=0.38$, $95 \% \mathrm{Cl}: 0.05-0.70, p=0.023$ ), but the quadratic effect was no longer significant (quadratic effect of depression, estimate: $-0.005,95 \% \mathrm{Cl}:-0.01-0.00, \mathrm{p}=0.23$ ).

Results of the regression model are shown in Table 2 and a plot of this relationship is shown in Fig. 1. Other factors associated with a higher barriers score were age and employment (Table 3). To assess this relationship using more flexible modeling, we also examined a spline regression model. Spline regression is a non-parametric technique that divides the datasets into intervals with different fits, which may yield better results depending on the nature of the data. Results for spline regression were relatively similar but with a slower taper off at higher depression scores (see supplementary material). Likelihood ratio tests showed that a model including a quadratic term was a better fit than a spline regression model. 
Table 2

Multivariate linear regression predicting the Barriers to Being Physically Active Quiz total score

\begin{tabular}{|c|c|c|c|}
\hline & Total Barrie & s Scale & \\
\hline Predictors & Estimates & $\mathrm{Cl}$ & $p$ \\
\hline (Intercept) & 29.48 & $21.76-37.20$ & $<0.001$ \\
\hline Age (10-year intervals) ${ }^{1}$ & -0.17 & $-0.26--0.08$ & $<0.001$ \\
\hline $\begin{array}{l}\text { Paid full or part-time } \\
\text { employment }\end{array}$ & 2.71 & $0.37-5.04$ & 0.024 \\
\hline Children living at home & -0.01 & $-2.52-2.50$ & 0.992 \\
\hline Never married ${ }^{2}$ & 0.18 & $-2.17-2.52$ & 0.881 \\
\hline Divorced/widowed ${ }^{2}$ & -0.49 & $-3.24-2.26$ & 0.725 \\
\hline Driving in past week & -0.54 & $-3.21-2.13$ & 0.690 \\
\hline Body mass index $\left(\mathrm{kg} / \mathrm{m}^{2}\right)$ & -0.08 & $-0.24-0.09$ & 0.363 \\
\hline CES-D Score (continuous) & 0.75 & $0.39-1.12$ & $<0.001$ \\
\hline Quadratic effect of depression & -0.02 & $-0.03--0.01$ & 0.002 \\
\hline
\end{tabular}

CES-D: Center for Epidemiological Studies Depression Scale. ${ }^{1}$ Age was divided into 10 -year intervals to increase interpretability. ${ }^{2}$ being married was the reference level.

\section{The Barriers to Being Physically Active Quiz Subscales}

Multivariate linear regression showed a significant relationship with depression scores for the social influence subscales (linear effect of depression, estimate $=0.03,95 \% \mathrm{Cl}: 0.00-0.06, p=0.027$, Table 3A), and the energy subscale (linear effect of depression, estimate $=0.04,95 \% \mathrm{Cl}: 0.01-0.07, p=0.017$, Table 3B). The subscales lack of resources, lack of skill and lack of willpower were all marginally significant and positively associated ( $p<0.01$; Supplementary materials). Models without quadratic effects were better fits. For the social influence scale and the energy scale, we removed 3 influential observations based on Cook's $d$. Results were similar when retaining influential observations in the model. Of note, the subscale results are in the context of an exploratory analysis and would not pass the threshold for multiple comparisons. 


\begin{tabular}{|c|c|c|c|}
\hline $3 A$. & Influence 0 & & \\
\hline Predictors & Estimates & $\mathrm{Cl}$ & $p$ \\
\hline (Intercept) & 3.87 & $2.17-5.57$ & $<0.001$ \\
\hline Age (10 year intervals) ${ }^{1}$ & -0.03 & $-0.05--0.01$ & 0.005 \\
\hline Paid full or part-time & -0.17 & $-0.69-0.34$ & 0.516 \\
\hline \multicolumn{4}{|l|}{ Employment } \\
\hline Children living at home & -0.03 & $-0.58-0.53$ & 0.928 \\
\hline Never married ${ }^{2}$ & -0.26 & $-0.79-0.26$ & 0.321 \\
\hline Divorced/widowed ${ }^{2}$ & 0.28 & $-0.33-0.88$ & 0.371 \\
\hline Driving in past week & -0.12 & $-0.72-0.48$ & 0.697 \\
\hline Body Mass Index $\left(\mathrm{kg} / \mathrm{m}^{2}\right)$ & 0.04 & $0.00-0.08$ & 0.03 \\
\hline CES-D scores (continuous) & 0.03 & $0.00-0.06$ & 0.027 \\
\hline 3B. & \multicolumn{3}{|l|}{ Energy } \\
\hline Predictors & Estimates & $\mathrm{Cl}$ & $p$ \\
\hline (Intercept) & 6.13 & $4.13-8.13$ & $<0.001$ \\
\hline Age (10-year intervals) ${ }^{1}$ & -0.06 & $-0.09--0.04$ & $<0.001$ \\
\hline Paid full or part-time & 1.59 & $0.99-2.18$ & $<0.001$ \\
\hline \multicolumn{4}{|l|}{ Employment } \\
\hline Children living at home & 0.08 & $-0.57-0.73$ & 0.81 \\
\hline Never married $^{2}$ & 0.47 & $-0.14-1.08$ & 0.135 \\
\hline Divorced/widowed ${ }^{2}$ & -0.02 & $-0.73-0.69$ & 0.955 \\
\hline Driving in past week & 0.07 & $-0.62-0.76$ & 0.84 \\
\hline Body Mass Index $\left(\mathrm{kg} / \mathrm{m}^{2}\right)$ & -0.01 & $-0.05-0.04$ & 0.784 \\
\hline CES-D scores (continuous) & 0.04 & $0.01-0.07$ & 0.017 \\
\hline
\end{tabular}

CES-D: Center for Epidemiological Studies Depression Scale. ${ }^{1}$ Age was divided into 10 -year intervals to increase interpretability. ${ }^{2}$ being married was the reference level. 


\section{Discussion}

We showed that higher depression scores were associated with higher physical activity barriers when adjusting for sociodemographic and clinical variables. There was a quadratic effect of depression on physical activity barriers, so that as CES-D scores increased, the positive relationship with physical activity barriers tapered off. These results indicate that depression level is a particularly important factor to consider when designing physical activity interventions for physically inactive women.

Our study shows that physical activity interventions for inactive women with depression diagnoses or symptoms may benefit from taking into account their greater perceived barriers to engaging in exercise. Many physical activity interventions still use a 'one size fits all' approach. For instance, a review paper illustrated that unique barriers to physical activity in mental health are usually not accounted for within behavior change theories used to design physical activity interventions(27). This may be one of the reasons that individuals with depression are less likely to engage in exercise programs, especially when they have a low baseline level of fitness(10). Previous work shows that physical activity interventions are more effective when they are tailored to subgroups like women and ethnic minorities(28). These findings suggest interventions may further increase their potential by tailoring depression levels as well.

\section{Most important barriers for physical activity}

For the sample as a whole, we identified that willpower, lack of time and lack of energy were the most significant barriers whereas injury and lack of skill were less often reported. This is consistent with previous research in physically inactive pregnant women and a community sample of Brazilian women, also using the same barriers scale(29, 30).

The Barriers to Being Physically Active Quiz subscales that were most associated with depression in a posthoc analysis were the Social Influence and Lack of Energy subscales. Although we assessed these relationships in an exploratory analysis, these barriers may be the driving factors behind the differences in total subscale scores.

The Social Influence subscale included statements such as: "None of my family members or friends like to do anything active, so I don't have a chance to exercise." and "I'm embarrassed about how I will look when I exercise with others". A lack of social support has been suggested as a risk factor for physical inactivity before(31), and our results show that this may be even more important in women with high depression scores. A review on physical activity barriers in depression (not specific to women) also identified other people's behaviors and a lack of social encouragement as important barriers, and social support as a facilitator(14). Other research found that having a family member who exercises, or who encourages exercise, motivates engaging in healthy behaviors(32). Our findings also suggest that selfconsciousness in social exercise-related situations (e.g. appearance towards others when exercising) may be an important factor discouraging women with higher depressive symptoms from physical activity. 
The Lack of Energy subscale includes statements like: "I'm too tired after work to catch up on exercise", and "I need the weekend to catch up on sleep". A lack of energy and increased fatigue are symptoms of depression. Low energy was also identified before as one of the most important barriers in men and women with depression(14). Of note, changes in physical activity may improve energy, and reduce the magnitude of perceived energy as a barrier(29).

Our posthoc results, though they need to be confirmed in future work, suggests the need for an emphasis on social influence and boosting energy to increase the effectiveness of physical activity promotion. Physical activity interventions could, for instance, integrate social support from family or friends, utilize peer-support or use community-based structures. Furthermore, exercise interventions can build-in graded exercise, personalized to a women's individual fitness levels to help slowly overcome feelings of fatigue(33). Future work should also quantify and integrate facilitators to exercise in women with high depressive symptoms. In mixed gender populations with depression, facilitators included having a reason for exercising, being able to identify the psychological benefits of exercise, having positive social support and integrating cognitive behavior change strategies(34).

Physical activity interventions are increasingly delivered in digital formats and via smartphones, using apps, and text-messaging and conversational agents. There is a growing interest in adaptive interventions, which alter their content based on the day-to-day behavior of individuals(35). We argue that physical activity interventions should both adapt to individuals' daily changing circumstances, and tailor their content to overcoming barriers of user subgroups.

\section{The quadratic effect of depression}

After a depression score (CES-D) of around 20, past the clinical cut-off for identifying individuals at risk for clinical depression ( $\geq 16$ ), physical activity barriers no longer increased with higher scores. We can't make finite conclusions about why this effect tapers off but there are multiple possible explanations. First, for participants with higher depression scores, physical activity may not be a priority and therefore they are less aware of their physical activity barriers. Another potential explanation is that the Barriers to Being Physically Active Quiz scale doesn't capture all barriers that are relevant when women reach more severe levels of depression. For instance, previous research(36) showed that in severe mental illness, low mood and stress are perceived as the most significant barriers for physical activity, followed by social support. In addition, in outpatients with depression, physical exertion was the most common reported PA barrier(37). The Barriers to Being Physically Active Quiz scale used in this study assess lack of energy, but it does not capture whether low mood, high stress or physical exhaustion prevent women from exercising. We recommend these questions be included in future versions.

One caveat here is that our data were sparse for very high depression scores, making these estimates less precise. Further, when we retained influential observations in the model the quadratic effect lost significance. Future work should assess differences in barriers between women with slightly elevated symptoms and clinically diagnosed depression.

\section{Age, employment and physical activity}


Another interesting finding was the inverse relationship between age and perceived physical activity barriers. In our posthoc analyses higher age was associated with lower barrier subscale scores, except for the injury and skills scales. These findings complement previous work in community samples showing that younger adults, both men and women, (25-44 years) report most physical activity barriers, and older adults (> 65 years) least(38). In line with our findings, earlier work has also identified lack of time and energy due to family and household responsibilities among the top barriers to physical activity for women(39). Older women may be less impacted by these responsibilities.

Finally, full-time or part-time employment was associated with higher barriers opposed to unemployment. Past work revealed that working women perceived lack of time and energy as most frequently reported barriers to physical activity(26). Future interventions may want to incorporate strategies for promoting physical activity into working hours to overcome these barriers for working women.

\section{Strengths \& Limitations}

We included a relatively large sample of diverse women. To our knowledge, this is one of the first studies to systematically examine the relationship between physical activity barriers and depressive symptoms in women using the instrument that was developed and validated by the $\operatorname{CDC}(14)$.

A limitation is that findings may be specific to our sample of female adults aged 25-65 years with relatively high levels of education from the San Francisco Bay Area. Our sample included fewer women with high depression scores, i.e. CESD $>20$, therefore the estimate of the relationship may be less reliable after these higher scores. In addition, our analyses are cross-sectional, thus don't allow us to understand the causal relationship between perceived barriers and depression. This should be explored in future work. Another limitation may be selection bias. Since we included women who signed up to participate in a physical activity intervention, the study participants might be more motivated to engage in physical activity. Further, women with higher depressive symptoms are less likely to participate in the study. This may in part explain the tapering off effects we observe for high depression scores.

\section{Conclusion}

In community dwelling women who enrolled in the MPED trial, higher depression scores were associated with higher physical activity barriers when correcting known confounding factors. In an exploratory posthoc analysis we identified social influence and lack of energy as particularly important physical activity barriers. Thus, addressing these barriers may improve an efficacy of physical activity interventions in women with high depressive symptoms. Further research, such as an RCT, is warranted.

\section{Abbreviations}

$\mathrm{BBAQ}=$ the Barriers to Being Active Quiz, BMI = Mody Mass Index, CES-D = Center for Epidemiological Studies Depression Scale. 


\section{Declarations}

Ethics approval: The study protocol was approved by the University of California, San Francisco (UCSF) Committee on Human Research and the mPED Data and Safety Monitoring Board (DSMB).

Consent to participate: All participants provided written consent prior to study enrollment.

Consent for publication: not applicable

Availability of data and materials: All data generated or analysed during this study are included in this published article [and its supplementary information files].

Competing of Interests: the authors report no competing interests.

Author contributions: YF designed and implemented the study and collected the data. CAF drafted the first version of the manuscript and conducted the quantitative analysis. TJH supervised the statistical analysis. AA assisted with interpreting the results of the analyses. All authors contributed to the writing of the final manuscript.

Funding: The project described was supported by the Award Number R01 HL104147 from the National Heart, Lung, and Blood Institute, by the American Heart Association, and by a grant (K24NR015812) from the National Institute of Nursing Research

Acknowledgements: Not applicable.

\section{References}

1. Tucker JM, Welk GJ, Beyler NK. Physical activity in US adults: compliance with the physical activity guidelines for Americans. American journal of preventive medicine. 2011;40(4):454-61.

2. KW Troiano RB, D Dodd, Masse LC, Tilert T, McDowell M.. Physical activity in the US measured by accelerometer. Med Sci Sports Exerc.40(1):181.

3. Kwak L, Berrigan D, Van Domelen D, Sjöström M, Hagströmer M. Examining differences in physical activity levels by employment status and/or job activity level: Gender-specific comparisons between the United States and Sweden. Journal of science and medicine in sport. 2016;19(6):482-7.

4. Sequeira S, Cruz C, Pinto D, Santos L, Marques A. Prevalence of barriers for physical activity in adults according to gender and socioeconomic status. British Journal of Sports Medicine. 2011;45(15):A18A9.

5. Arango EF, Patiño FA, Quintero MA, Arenas MM. Levels of physical activity, barriers, and stage of change in an urban population from a municipality in Colombia. Colombia Médica. 2011;42(3):35261.

6. Booth ML, Bauman A, Owen N. Perceived barriers to physical activity among older Australians. Journal of aging and physical activity. 2002;10(3):271-80. 
7. Juarbe T, Turok XP, Pérez-Stable EJ. Perceived benefits and barriers to physical activity among older Latina women. Western journal of nursing research. 2002;24(8):868-86.

8. Kuehner $\mathrm{C}$. Why is depression more common among women than among men? The Lancet Psychiatry. 2017;4(2):146-58.

9. Choi KW, Chen C-Y, Stein MB, Klimentidis YC, Wang M-J, Koenen KC, et al. Assessment of Bidirectional Relationships Between Physical Activity and Depression Among Adults: A 2-Sample Mendelian Randomization Study. JAMA Psychiatry. 2019.

10. Blumenthal JA, Smith PJ, Hoffman BM. Is exercise a viable treatment for depression? ACSM's health \& fitness journal. 2012;16(4):14.

11. Schuch FB, Vancampfort D, Richards J, Rosenbaum S, Ward PB, Stubbs B. Exercise as a treatment for depression: a meta-analysis adjusting for publication bias. Journal of psychiatric research. 2016;77:42-51.

12. Mammen G, Faulkner G. Physical activity and the prevention of depression: a systematic review of prospective studies. American journal of preventive medicine. 2013;45(5):649-57.

13. Cla T. Time to tackle the physical activity gender gap. Health (Irvine Calif). 2018;6:e1077-86.

14. Glowacki K, Duncan MJ, Gainforth H, Faulkner G. Barriers and facilitators to physical activity and exercise among adults with depression: A scoping review. Mental Health and Physical Activity. 2017;13:108-19.

15. Schoeny ME, Fogg L, Buchholz SW, Miller A, Wilbur J. Barriers to physical activity as moderators of intervention effects. Preventive medicine reports. 2017;5:57-64.

16. Reed JL, Prince SA, Elliott CG, Mullen K-A, Tulloch HE, Hiremath S, et al. Impact of workplace physical activity interventions on physical activity and cardiometabolic health among working-age women: a systematic review and meta-analysis. Circulation: Cardiovascular Quality and Outcomes. 2017;10(2):e003516.

17. Lambert JD, Greaves CJ, Farrand P, Price L, Haase AM, Taylor AH. Web-Based Intervention Using Behavioral Activation and Physical Activity for Adults With Depression (The eMotion Study): Pilot Randomized Controlled Trial. Journal of medical Internet research. 2018;20(7):e10112-e.

18. Murray JM, Brennan SF, French DP, Patterson CC, Kee F, Hunter RF. Effectiveness of physical activity interventions in achieving behaviour change maintenance in young and middle aged adults: a systematic review and meta-analysis. Social Science \& Medicine. 2017;192:125-33.

19. Fukuoka Y, Komatsu J, Suarez L, Vittinghoff E, Haskell W, Noorishad T, et al. The mPED randomized controlled clinical trial: applying mobile persuasive technologies to increase physical activity in sedentary women protocol. BMC public health. 2011;11(1):1-8.

20. Fukuoka Y, Lindgren TG, Mintz YD, Hooper J, Aswani A. Applying natural language processing to understand motivational profiles for maintaining physical activity after a mobile app and accelerometer-based intervention: the MPED randomized controlled trial. JMIR mHealth and uHealth. 2018;6(6):e10042. 
21. Fukuoka Y, Haskell W, Lin F, Vittinghoff E. Short-and Long-term Effects of a Mobile Phone App in Conjunction With Brief In-Person Counseling on Physical Activity Among Physically Inactive Women: The mPED Randomized Clinical Trial. JAMA network open. 2019;2(5):e194281-e.

22. Zhou M, Fukuoka Y, Goldberg K, Vittinghoff E, Aswani A. Applying machine learning to predict future adherence to physical activity programs. BMC medical informatics and decision making. 2019;19(1):169.

23. Fukuoka Y, Haskell W, Vittinghoff E. New insights into discrepancies between self-reported and accelerometer-measured moderate to vigorous physical activity among women-the mPED trial. BMC public health. 2016;16(1):761.

24. Weissman MM, Sholomskas D, Pottenger M, Prusoff BA, Locke BZ. Assessing depressive symptoms in five psychiatric populations: a validation study. Am J Epidemiol. 1977;106(3):203-14.

25. Barriers to Being Physically Active Quiz. US Department of Health and Human Services. 1999.

26. Mariam A-A, Mazin H. Working experience and perceived physical activity and exercise barriers. Sport Mont. 2019;17(2):47-52.

27. Rebar AL, Taylor A. Physical activity and mental health; it is more than just a prescription. Mental Health and Physical Activity. 2017;13:77-82.

28. Bock C, Jarczok MN, Litaker D. Community-based efforts to promote physical activity: a systematic review of interventions considering mode of delivery, study quality and population subgroups. Journal of science and medicine in sport. 2014;17(3):276-82.

29. Choi J, hyeon Lee J, Vittinghoff E, Fukuoka Y. mHealth physical activity intervention: a randomized pilot study in physically inactive pregnant women. Maternal and child health journal. 2016;20(5):1091-101.

30. Herazo-Beltrán Y, Pinillos Y, Vidarte J, Crissien E, Suarez D, García R. Predictors of perceived barriers to physical activity in the general adult population: a cross-sectional study. Brazilian journal of physical therapy. 2017;21(1):44-50.

31. Scarapicchia TMF, Amireault S, Faulkner G, Sabiston CM. Social support and physical activity participation among healthy adults: A systematic review of prospective studies. International Review of Sport and Exercise Psychology. 2017;10(1):50-83.

32. Ashida S, Wilkinson AV, Koehly LM. Social influence and motivation to change health behaviors among Mexican-origin adults: implications for diet and physical activity. American Journal of Health Promotion. 2012;26(3):176-9.

33. Marin $H$, Menza MA. Specific treatment of residual fatigue in depressed patients. Psychiatry (Edgmont). 2004;1(2):12.

34. Rogerson MC, Murphy BM, Bird S, Morris T. "I don't have the heart": a qualitative study of barriers to and facilitators of physical activity for people with coronary heart disease and depressive symptoms. International Journal of Behavioral Nutrition and Physical Activity. 2012;9(1):1-9.

35. Nahum-Shani I, Smith SN, Spring BJ, Collins LM, Witkiewitz K, Tewari A, et al. Just-in-Time Adaptive Interventions (JITAls) in Mobile Health: Key Components and Design Principles for Ongoing Health 
Behavior Support. Annals of behavioral medicine: a publication of the Society of Behavioral Medicine. 2018;52(6):446-62.

36. Firth J, Rosenbaum S, Stubbs B, Gorczynski P, Yung AR, Vancampfort D. Motivating factors and barriers towards exercise in severe mental illness: a systematic review and meta-analysis.

Psychological medicine. 2016;46(14):2869-81.

37. Monteiro FC, Schuch FB, Deslandes AC, Vancampfort D, Mosqueiro BP, Messinger MF, et al. Perceived barriers, benefits and correlates of physical activity in outpatients with Major Depressive Disorder: A study from Brazil. Psychiatry Research. 2020;284:112751.

38. Hickey ME, Mason SE. Age and gender differences in particpation rates, motivators for, and barriers to exercise. Modern Psychological Studies. 2017;22(2):3.

39. Nezami BT, Jakicic JM, Lang W, Davis K, Tate DF. Examining barriers, physical activity, and weight change among parents and nonparents in a weight loss intervention. Obesity Science \& Practice. 2020.

\section{Figures}




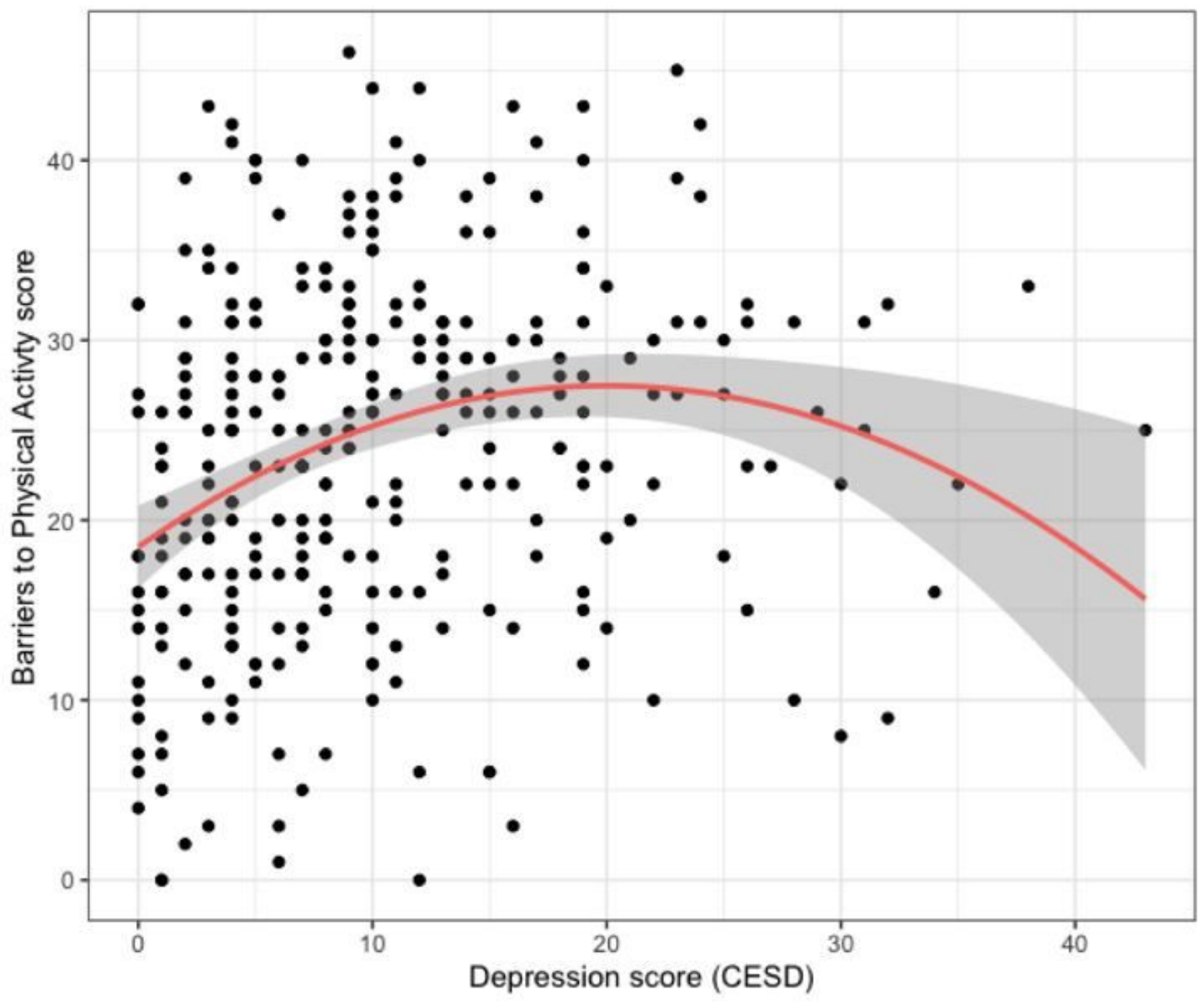

Figure 1

Quadratic relationship between physical activity barriers score and depression scores.

\section{Supplementary Files}

This is a list of supplementary files associated with this preprint. Click to download.

- Supplementarymaterialbarriers12.30.docx 